\title{
Long-term follow-up after vitrectomy to treat idiopathic full-thickness macular holes: visual acuity and macular complications
}

This article was published in the following Dove Press journal:

Clinical Ophthalmology

8 August 2012

Number of times this article has been viewed

\author{
Hirokazu Sakaguchi' \\ Masahito Ohji ${ }^{2}$ \\ Yusuke Oshima' \\ Yasushi Ikuno' \\ Fumi Gomi' \\ Naoyuki Maeda' \\ Motohiro Kamei' \\ Shunji Kusaka ${ }^{3}$ \\ Kohji Nishida' \\ 'Department of Ophthalmology, \\ Osaka University Graduate School \\ of Medicine, Suita, ${ }^{2}$ Department of \\ Ophthalmology, Shiga University of \\ Medical Science, Otsu, ${ }^{3}$ Department \\ of Ophthalmology, Sakai Hospital \\ Kinki University Faculty of Medicine, \\ Sakai, Japan
}

Correspondence: Hirokazu Sakaguchi Department of Ophthalmology, Osaka University Graduate School of Medicine, 2-2 Yamadaoka, E-7, Suita,

Osaka 565-087I, Japan

Tel +8I 668793456

Fax +8I 668793458

Email sakaguh@ophthal.med.osaka-u.ac.jp
Background: To assess time-course changes in best-corrected visual acuity (BCVA) up to 5 years after vitrectomy to treat idiopathic full-thickness macular holes (MHs) and identify the relationship of the changes to postoperative complications.

Methods: Twenty-three consecutive patients with an idiopathic MH underwent vitrectomy without adjuvant treatment. BCVA and complications were assessed postoperatively.

Results: Twenty-two of $23(95.7 \%)$ MHs closed after the first surgery, with a final anatomic success rate of $100 \%$. The time course of the BCVA was analyzed in 20 cases in which data were obtained for over 5 years. The BCVA improved by 0.43 logarithm of the minimum angle of resolution ( $\log$ MAR) unit 6 months postoperatively $(P<0.001)$ and continuously improved by $0.05,0.06$, and $0.07 \log$ MAR units between 6 months and 1 year, 1 year and 3 years (by 0.11 $\log$ MAR unit between 6 months and 3 years; $P=0.049)$, and 3 years and 5 years $(P=0.018)$ postoperatively, respectively. Macular complications developed in seven (35\%) of the 20 cases; the mean BCVA at 5 years in these cases was significantly $(P<0.001)$ worse than in cases without complications.

Conclusion: The BCVA might improve gradually for 5 years after vitrectomy to treat MHs. However, the macular complications that can develop postoperatively could limit that possibility.

Keywords: macula hole, time course, vitrectomy, retina, visual acuity, complication

\section{Introduction}

Kelly and Wendel, ${ }^{1}$ who first reported the effectiveness of vitrectomy for treating idiopathic macular holes (MHs), showed that best-corrected visual acuity (BCVA) might improve with anatomic closure of the MHs. Several methods, including removal of the internal limiting membrane (ILM) and use of adjuvants (transforming growth factor-beta 2 [TGF- $\beta_{2}$ ] or autologous serum), were developed to improve the closure rate. ${ }^{2-7}$

Although the effectiveness of vitrectomy for treating MHs has been reported frequently, the follow-up periods were largely limited to 6 or 12 months, and most studies did not include a time-course analysis.

A few studies have reported follow-up periods exceeding 3 years after vitrectomy for idiopathic MHs. Leonard et $\mathrm{al}^{8}$ reported the visual acuity (VA) time course up to 3 years and continuous VA improvements after vitrectomy with TGF- $\beta_{2}$ and without ILM removal for MHs; however, only $73 \%$ of the patients were followed for 3 years. Scott et $\mathrm{al}^{9}$ performed vitrectomy with TGF- $\beta_{2}$ in $91 \%$ of the cases without ILM removal and reported the final VA after more than 5 years and the best VA. Haritoglou et al ${ }^{10}$ 
reported results after more than 3 years following vitrectomy with ILM peeling. Neither of these latter two studies included a time-course analysis.

Regarding complications, Leonard et $\mathrm{al}^{8}$ reported that one $\mathrm{MH}$ reopened after cataract surgery and no retinal detachments developed. Scott et $\mathrm{al}^{9}$ found that choroidal neovascularization associated with age-related macular degeneration and retinal detachment resulted in decreased VA. Haritoglou et $\mathrm{al}^{10}$ reported that no $\mathrm{MHs}$ opened again after the initial closure and there was no cellular proliferation in the macular area.

The current study assessed the time-course changes in BCVA and postoperative complications during follow-up for 5 years in patients with a $\mathrm{MH}$ after vitrectomy.

\section{Methods}

\section{Patients}

Twenty-three consecutive patients who presented to the Osaka University Hospital with full-thickness stage 2-4 idiopathic $\mathrm{MH}$ were enrolled in this uncontrolled interventional case study. The surgeries were performed from February 1998 to April 1999.

Patients were excluded if symptoms persisted for longer than 1 year, if they had undergone a previous posterior surgery, or had glaucoma, retinal vein occlusion, high myopia exceeding -6.0 diopters, or systemic diseases that precluded maintaining a facedown position.

Preoperative data included patient age, sex, affected eye, spherical equivalent refraction, preoperative symptom duration, $\mathrm{MH}$ diameter in disc diameters (DD), $\mathrm{MH}$ stage (range, 2-4), lens status (phakia, pseudophakia, aphakia), and BCVA (Table 1). MH stage was based on Gass's classification $^{11}$ and confirmed by biomicroscopy and/or

Table I Preoperative characteristics $(n=23)$

\begin{tabular}{lll}
\hline Age, years (range) & $63.6 \pm 5.8$ & $(52-72)$ \\
Men (\%) & 14 & $(60.9)$ \\
Right eye (\%) & 9 & $(39.1)$ \\
Preoperative & $3.1 \pm 2.3$ & $(1-9)$ \\
symptom duration (months) & & \\
Refraction (D) & $-0.44 \pm 2.23$ & $(-5.25$ to 3.5$)$ \\
Phakic eyes (\%) & 23 & $(100.0)$ \\
MH diameter (DD) & $0.21 \pm 0.11$ & $(0.05-0.46)$ \\
& & $($ data missing from \\
& $13(56.5 \%) / 7$ & four eyes) \\
No stage 2/3/4 MHs (\%) & $(30.5 \%) / 3(13.0 \%)$ & \\
BCVA (mean) & 0.20 & $(0.04-0.8)$
\end{tabular}

Note: Refraction = spherical equivalent refraction.

Abbreviations: D, diopters; DD, disc diameter; $\mathrm{MH}$, macular hole; BCVA, bestcorrected visual acuity. optical coherence tomography (OCT). $\mathrm{MH}$ diameter was calculated by averaging the vertical and horizontal lengths of MHs compared to the disc. BCVA was measured using the Landolt C VA chart. Manual refraction was performed when acuity was measured in this trial.

\section{Surgical techniques}

A standard three-port pars plana vitrectomy was performed by four surgeons. A posterior vitreous detachment (PVD) was created with active aspiration in cases without complete PVD. Intentional ILM peeling was not performed. If the vitreous cortex around the MHs was observed, it was gently removed using a diamond-dusted membrane scraper (DDMS) (Synergetic, St Charles, MO). ${ }^{12,13}$ The surface of the retina just around the MHs was gently scratched with DDMS, and the vitreous cortex attached to the edge of MHs was removed. Epiretinal membranes (ERMs) were removed if there were apparent ERMs. Combined phacoemulsification and intraocular lens implantation were performed after the patients received an explanation that progression of nuclear sclerosis after vitrectomy is not preventable in patients exceeding 50 years. ${ }^{14,15}$

Adjuvants or intravitreal injections of triamcinolone were not administered during any procedures. After fluid-air exchange, the air was exchanged for $12 \%-16 \%$ perfluoropropane. Patients remained facedown from immediately postoperatively for about 2 weeks. All patients provided written informed consent before any procedures were performed.

\section{Main outcomes}

Postoperative data recorded included BCVA, macular status (open/closed), and postoperative complications. BCVA data at 6 months and 1, 3, and 5 years postoperatively were analyzed in this study. BCVA measured at 6 months \pm 1 month, 1 year \pm 2 months, 3 years \pm 2 months, and 5 years \pm 2 months postoperatively was collected. A fundus examination and/or OCT was performed to detect macular changes and other complications postoperatively. Any intraoperative complications that developed were recorded.

To remove the influence of cataract surgery on the improvement of visual acuity, the relationship between cataract surgery and visual acuity was also analyzed.

Statistical analysis was performed using statistical analysis software (SigmaStat version 2.0; IBM, Armonk, NY). The data are expressed as the mean \pm standard deviation. BCVA levels were converted to the logarithm of the minimum angle of resolution (logMAR) equivalents and analyzed. Mean BCVAs at each time point were compared using the 
paired $t$-test, and $t$-tests were performed between the groups. Fisher's exact test was performed to analyze the proportions between each group. $P<0.05$ was considered significant.

\section{Results \\ Follow-up}

Data were obtained for more than 5 years (range, 61-100 months; mean, $83.4 \pm 10.5$ months) in 20 of 23 cases.

Preoperative BCVA levels in these cases ranged from 0.04 to 0.8 (mean, 0.19 ) in Landolt C VA. The mean patient age was $62.5 \pm 5.3$ years, and the refractive error was $-0.44 \pm 2.23$ diopters. The mean MH diameter was $0.21 \pm 0.11 \mathrm{DD}$ (range, $0.05-0.46$ ). Ten, seven, and three eyes had stage 2, 3, and $4 \mathrm{MHs}$, respectively. All cases were phakic.

Three patients who had a stage $2 \mathrm{MH}$ preoperatively could not keep their follow-up appointments because of new systemic diseases or relocation to a distant town. Those MHs closed after the first vitrectomy. Preoperative BCVA was 0.3 in all eyes, and BCVA improved to 0.5 at 1 year in one case, to 0.7 at 1 year in another case, and to 0.8 at 1 year and 1.0 at 3 years in the third case. No postoperative complications developed during the follow-up periods $(1,1$, and 3 years, respectively).

\section{Surgery}

Combined cataract surgery was performed in 18 (78.3\%) cases. A PVD was created with active aspiration in 20 of 23 cases. Two cases underwent ERM removal, and the vitreous cortex around the MHs was removed using a DDMS in twelve of 23 cases. ILM peeling was not performed, although the DDMS might have scratched the ILM surface just around the MHs. Mean surgical time was $61.6 \pm 13.4$ minutes.

MHs closed in 22 (95.7\%) of the 23 eyes postoperatively. Another vitrectomy was performed 2 months after the first surgery in one patient (age, 55 years) in whom the MH did not close after the first surgery; successful closure was achieved after the second surgery. Preoperative BCVA was 0.1 , refractive error was -0.25 diopter, preoperative duration of symptoms was 9 months, and $\mathrm{MH}$ diameter was $0.46 \mathrm{DD}$.

\section{Postoperative BCVA time course}

The time course of the BCVA was analyzed in the 20 cases up to 5 years (Figure 1). Mean BCVA improved continuously up to 5 years, even after 6 months. BCVAs at 6 months and 1,3 , and 5 years postoperatively ranged from 0.1 to 1.0 (mean, 0.50 ), 0.1 to 1.2 (mean, 0.56 ), 0.2 to 1.2 (mean, 0.64 ), and 0.2 to 1.2 (mean, 0.75 ) in Landolt C VA, respectively.

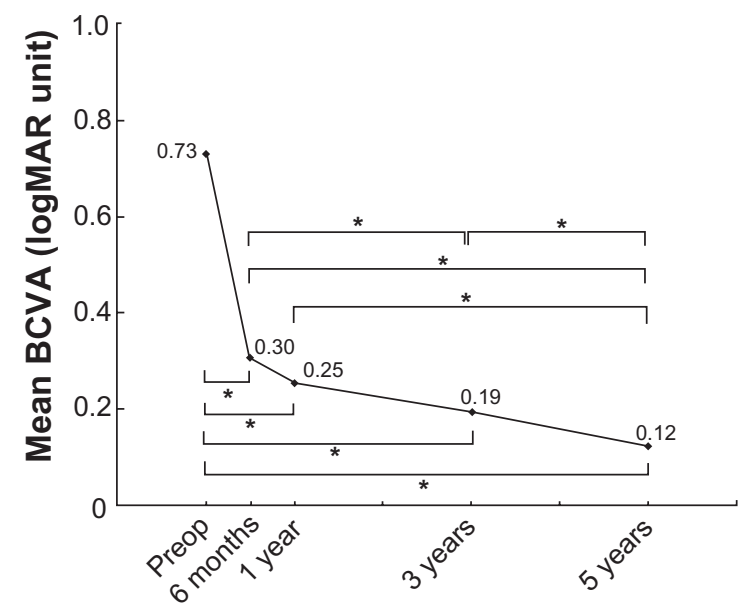

Follow-up period

Figure I Time-course changes in mean BCVA (logMAR units).

Notes: The BCVA levels in Landolt $C$ chart were converted to the logarithm of the minimum angle of resolution (logMAR) equivalents and analyzed. Mean BCVA improved continuously up to 5 years, even after 6 months. Asterisks indicate a significant difference.

Abbreviations: BCVA, best-corrected visual acuity; Preop, preoperative; logMAR, logarithm of the minimum angle of resolution.

BCVA improved by $0.43 \log$ MAR unit 6 months postoperatively compared with preoperatively $(P<0.001)$. BCVA improved by $0.05,0.06$, and $0.07 \log$ MAR units between 6 months and 1 year, 1 year and 3 years (by 0.11 $\log$ MAR unit between 6 months and 3 years; $P=0.049$ ), and 3 years and 5 years $(P=0.018)$ postoperatively, respectively. Therefore, BCVA improved more than $0.05 \log$ MAR unit between time points even after 6 months postoperatively. The largest proportion of the improvement occurred within the initial 6 months after the surgeries. There were also significant differences between BCVAs at 6 months and 5 years $(P=0.001)$ and between 1 year and 5 years $(P=0.002)$ (Figure 1).

Figure 2 shows the distribution of BCVA at each time point. The percentage of cases in which BCVA was

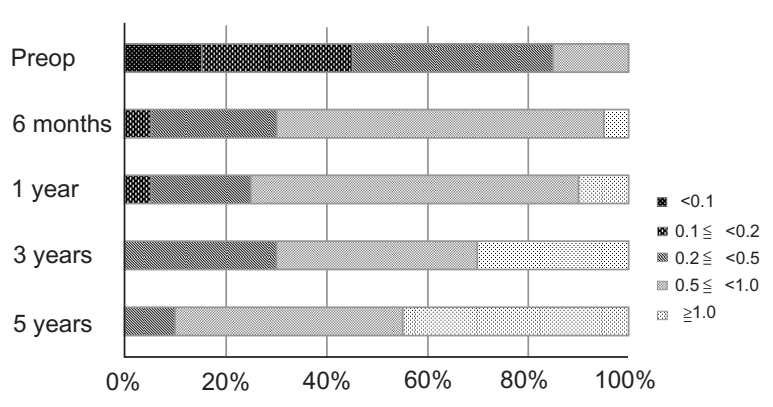

Figure 2 Distribution of BCVA values at each time point.

Notes: The percentage of cases with BCVA of 1.0 or better gradually increased and reached $45 \%$. No case had BCVA worse than 0.15 years postoperatively. Abbreviations: BCVA, best-corrected visual acuity; Preop, preoperative. 
1.0 or better in Landolt C VA increased during follow-up and reached $45 \%$ (nine cases) at 5 years postoperatively. The percentage of cases in which BCVA was 0.5 or higher reached 90\% (18 cases). No case had BCVA below 0.1 at 5 years. BCVA improved three or more lines in 75\% (15/20) of cases 5 years postoperatively.

BCVA values in the case in which the $\mathrm{MH}$ closed after the second surgery were $0.1,0.4,0.4,0.7$, and 0.6 in Landolt CVA preoperatively, at 6 months, and 1, 3, and 5 years postoperatively, respectively.

\section{Postoperative complications and BCVA}

No holes recurred in any eyes during the follow-up period. No retinal detachments or tears developed intraoperatively or postoperatively.

Five of the 20 cases that did not undergo simultaneous cataract surgery combined with vitrectomy developed cataracts postoperatively. Macular complications developed postoperatively: cystoid macular edema $(\mathrm{CME})(\mathrm{n}=1)$, chorioretinal degeneration $(n=1)$, and epiretinal proliferation $(n=5)$ in seven of the 20 cases during follow-up to 5 years (Table 2). No other complications developed intraoperatively or postoperatively.

CME was observed 6 months after vitrectomy in one eye and required another vitrectomy with ILM peeling without adjuvants. The BCVA in this case changed from 0.3 preoperatively to 0.5 in Landolt $\mathrm{C}$ VA at 5 years postoperatively.

Chorioretinal degeneration with depigmentation of the retinal pigment epithelium developed 2 months postoperatively in the foveal area of one eye. BCVA was 0.05 preoperatively, improved to 0.16 months postoperatively, and then varied between 0.1 and 0.2. BCVA at 5 years was 0.2 in Landolt C VA. This case had a stage $4 \mathrm{MH}$ and closed successfully.

Table 2 Postoperative complications

\begin{tabular}{lll}
\hline $\begin{array}{l}\text { Complication } \\
\text { (number of cases) }\end{array}$ & Onset after surgery & Treatment \\
\hline Cataract $(n=5)$ & $\begin{array}{l}0.5-2.5 \\
(\text { mean } 1.4 \pm 0.74) \text { years }\end{array}$ & Cataract surgery \\
$\begin{array}{l}\text { Cystoid macular } \\
\text { edema }(n=I)\end{array}$ & $\begin{array}{l}\text { PPV with ILM } \\
\text { removal }\end{array}$ \\
$\begin{array}{l}\text { Chorioretinal } \\
\text { degeneration }(n=1)\end{array}$ & None \\
$\begin{array}{l}\text { Epiretinal } \\
\text { proliferation }(n=1)\end{array}$ & $\begin{array}{l}\text { I.5-5 } \\
(\text { mean } 2.7 \pm 1.4) \text { years }\end{array}$ & None \\
\hline
\end{tabular}

Note: Cataract surgery refers to phacoemulsification and intraocular lens implantation. Abbreviations: PPV, pars plana vitrectomy; ILM, internal limiting membrane.
BCVAs in five cases in which epiretinal proliferation developed within the 5 years of follow-up changed from 0.04 , $0.3,0.3,0.4$, and 0.5 preoperatively to $0.6,0.5,0.7,0.4$, and 1.05 years postoperatively in Landolt C VA, respectively. The first apparent epiretinal proliferation was found in one case 1.5 years postoperatively. Two of the five cases with postoperative epiretinal proliferation were those that underwent ERM removal during vitrectomy for a $\mathrm{MH}$.

Mean BCVA was 0.93 in Landolt C VA in cases without complications 5 years postoperatively. Mean BCVA was 0.51 in cases with complications 5 years postoperatively. The difference between these two groups was significant $(P=0.0009)$, although there was no difference between the two groups preoperatively $(P>0.05)$. In cases without complications, $84.6 \%$ had a BCVA improvement of $0.3 \log$ MAR unit or more 5 years postoperatively, compared with $57.1 \%$ cases with complications. The percentage of cases without complications with BCVA of 1.0 or higher increased gradually and reached $61.5 \% 5$ years postoperatively. However, the percentage of cases with complications was $14.3 \%$.

\section{Cataract surgery and BCVA}

Combined cataract surgery was performed in 18 (78.3\%) of 23 cases. Data were obtained for more than 5 years in 15 of the 18 cases. Mean BCVAs in these cases were 0.15 (range, 0.04-0.5), 0.45 (range, 0.1-1.0), 0.54 (range, 0.1-1.2), 0.56 (range, 0.2-1.2), and 0.67 (range, 0.2-1.2) in Landolt C VA, preoperatively, at 6 months, 1, 3, and 5 years postoperatively, respectively. There were significant differences between BCVAs at 6 months and 5 years $(P=0.011)$, between 1 year and 5 years $(P=0.047)$, and between 3 years and 5 years $(P=0.047)$.

Phacoemulsification and intraocular lens implantation were performed in two eyes within 1 year after vitrectomy, in two eyes between 1 and 2 years, and in one eye between 2 and 3 years among the cases in which simultaneous cataract surgery combined with vitrectomy was not performed. Mean BCVAs in these cases were 0.8 (range, 0.6-1.0), 0.82 (range, 0.6-1.0), 0.86 (range, 0.5-1.0), and 1.08 (range, 1.0-1.2) in Landolt C VA at 6 months and 1, 2, and 3 years after cataract surgery, respectively. There was a significant difference between BCVAs at 6 months and at 3 years $(P=0.012)$ and between 1 year and 3 years $(P=0.015)$ after cataract surgery.

No postoperative macular complications developed in the cases that did not undergo simultaneous cataract surgery. All five cases $(100 \%)$ treated with vitrectomy followed by cataract surgery achieved BCVA of 1.0 or better, while four 
of 15 cases $(26.7 \%)$ treated with vitrectomy combined with simultaneous cataract surgery achieved that level of BCVA ( $P=0.008$, Fisher's exact test), although the preoperative VA in these two groups did not differ significantly $(P>0.05)$.

\section{Discussion}

We investigated if BCVA continues to improve to a minimum of 5 years after vitrectomy for MHs. In a 2-year time-course study, Ezra and Gregor ${ }^{16}$ reported that final BCVA was better than at 1 year. They theorized that this improvement resulted from cataract surgery having been performed, in $72.2 \%$ of the cases, between 1 and 2 years after vitrectomy. In the current study, cataract surgery was performed after vitrectomy in $25 \%$ of our cases, and the surgery was performed simultaneously with vitrectomy in $75 \%$. The percentage of our cases that underwent cataract surgery after vitrectomy was lower than that reported by Ezra and Gregor. ${ }^{16}$ In the current study, cataract surgery was performed in five eyes with time periods within 1 year, between 1 and 2 years, and between 2 and 3 years after vitrectomy. Thus, all cataract surgeries were performed before 3 years after vitrectomy. Mean BCVA in all of the current cases significantly increased, even after 3 years postoperatively. This result suggested that reasons other than cataract surgery were responsible for the BCVA improvement. Mean BCVA for the 15 cases in which combined cataract surgery with vitrectomy was performed increased significantly postoperatively. Moreover, mean BCVA for the five cases in which cataract surgery was performed after vitrectomy also significantly improved in the time course immediately after cataract surgery. These results also suggested that reasons other than cataract surgery were responsible for the BCVA improvement. Foveal function with reconstruction of the foveal microstructure might have been a factor. We reported previously that reconstruction of the foveal microstructure might improve visual function ${ }^{17}$ during a 1-year follow-up period. The foveal microstructure might gradually improve even after 1 year postoperatively, with resultant improvement of visual function. In this study, the foveal microstructure was not examined because timedomain OCT, which was used in this study, could not show the details of foveal microstructure, such as inner segment/ outer segment junction. That was one of the limitations in this study.

BCVA improved by $0.43 \log$ MAR unit 6 months postoperatively compared with preoperatively. BCVA improved by $0.05,0.06$, and $0.07 \log$ MAR units between 6 months and 1 year, 1 year and 3 years, and 3 years and 5 years postoperatively, respectively. Thus, BCVA improved more than $0.05 \log$ MAR unit between time points even after 6 months postoperatively up to 5 years postoperatively. Meanwhile, the largest proportion of the improvement occurred within the initial 6 months after the surgeries. Wittich et $\mathrm{al}^{18}$ examined acuity recovery rate after MH surgery, and using hierarchical linear modeling with linear and curvilinear regression analysis showed that most recuperation occurred during the initial 6 months after $\mathrm{MH}$ surgery (follow-up, 6-23 months). The data corresponded to our result.

BCVAs of 0.5 or better and 1.0 or better in Landolt C VA increased gradually during follow-up and reached $90 \%$ and $45 \%$ at 5 years postoperatively, respectively, in the current study. Scott et $\mathrm{al}^{9}$ reported a final VA of 20/40 or higher in $58 \%$ of cases, which was lower than in the current study. One reason for the difference was the preoperative status of the study cases; the duration between the onset of subjective symptoms and surgery in their study ranged from 1.1 to 93.8 months, compared with 1-9 months in the current study. Another possibility is the surgical method; most of the cases of Scott et $\mathrm{al}^{9}$ included a TGF- $\beta$ protocol, which might have affected the long-term results. We did not use adjuvants in our cases.

In the current study, the rate of $\mathrm{MH}$ closure exceeded $95 \%$ after the first surgery, and we achieved a $100 \%$ final closure rate. The $\mathrm{MH}$ closure rates after vitrectomy without ILM peeling have ranged from $57.7 \%$ to $89 \%$. ${ }^{1,2,5,9,19}$ The rate in the current study was relatively higher than in those reports and the same in cases with ILM peeling or adjuvants, which also might have resulted from differences in preoperative status. Another possibility is that we used a DDMS intraoperatively. ${ }^{12,13}$ With the DDMS, the surface of the ILM can be scratched, and a focal area of ILM might be peeled.

Several complications developed after vitrectomy for idiopathic $\mathrm{MHs}$ in the current study during a long follow-up period that limited the improvement in visual function after $\mathrm{MH}$ closure. CME and chorioretinal degeneration developed within 1 year postoperatively, and BCVA did not reach 1.0 at 5 years postoperatively. Epiretinal proliferation might have resulted from the long-term response caused by injury to a portion of the Müller cells or inflammation intraoperatively and postoperatively and developed gradually in $25 \%$ of our cases. BCVA reached 1.0 in only one case with epiretinal proliferation. ERMs could have prevented BCVA improvement after $\mathrm{MH}$ closure. Most surgeons now remove the ILM during MH surgery. This procedure might minimize the recurrence of idiopathic ERMs. ${ }^{20}$ Thus this procedure 
may prevent epiretinal formation in the long term after vitrectomy to treat $\mathrm{MH}$. In fact, Haritoglou et $\mathrm{al}^{10}$ reported no ERM formation during a follow-up period that exceeded 36 months after vitrectomy with ILM removal for MHs. ILM removal might improve the rate of $\mathrm{MH}$ closure and prevent ERM formation after vitrectomy for MHs.

Analysis of the time-course changes in BCVA and postoperative complications up to 5 years after vitrectomy for consecutive $\mathrm{MH}$ cases suggested the possibility of gradual improvement in visual function and the limitations of the improvement if macular complications occur. A randomized control study should evaluate the effectiveness of ILM removal during vitrectomy for MHs to prevent ERM formation as a macular complication.

\section{Acknowledgments}

The authors are grateful for the leadership and experience of the late Professor Yasuo Tano, Chairman of the Department of Ophthalmology, Osaka University Graduate School of Medicine.

\section{Disclosure}

The authors have no proprietary interest in any aspect of this report. This research received no specific grant from any funding agency in the public, commercial or not-for-profit sectors.

\section{References}

1. Kelly NE, Wendel RT. Vitreous surgery for idiopathic macular holes. Results of a pilot study. Arch Ophthalmol. 1991;109:654-659.

2. Tognetto D, Grandin R, Sanguinetti G, et al. Internal limiting membrane removal during macular hole surgery: results of a multicenter retrospective study. Ophthalmology. 2006;113:1401-1410.

3. Da Mata AP, Burk SE, Foster RE, et al. Long-term follow-up of indocyanine green-assisted peeling of the retinal internal limiting membrane during vitrectomy surgery for idiopathic macular hole repair. Ophthalmology. 2004;111:2246-2253.

4. Kadonosono K, Itoh N, Uchio E, Nakamura S, Ohno S. Staining of internal limiting membrane in macular hole surgery. Arch Ophthalmol. 2000;118:1116-1118.

5. Sheidow TG, Blinder KJ, Holekamp N, et al. Outcome results in macular hole surgery: an evaluation of internal limiting membrane peeling with and without indocyanine green. Ophthalmology. 2003;110:1697-1701.
6. Kusaka S, Sakagami K, Kutsuna M, Ohashi Y. Treatment of fullthickness macular holes with autologous serum. Jpn J Ophthalmol. 1997;41:332-338.

7. Thompson JT, Smiddy WE, Williams GA, et al. Comparison of recombinant transforming growth factor-beta-2 and placebo as an adjunctive agent for macular hole surgery. Ophthalmology. 1998;105: 700-706.

8. Leonard RE 2nd, Smiddy WE, Flynn HW Jr, Feuer W. Long-term visual outcomes in patients with successful macular hole surgery. Ophthalmology. 1997;104:1648-1652.

9. Scott IU, Moraczewski AL, Smiddy WE, Flynn HW Jr, Feuer WJ. Longterm anatomic and visual acuity outcomes after initial anatomic success with macular hole surgery. Am J Ophthalmol. 2003;135:633-640.

10. Haritoglou C, Reiniger IW, Schaumberger M, Gass CA, Priglinger SG, Kampik A. Five-year follow-up of macular hole surgery with peeling of the internal limiting membrane: update of a prospective study. Retina. 2006;26:618-622.

11. Gass JD. Idiopathic senile macular hole. Its early stages and pathogenesis. Arch Ophthalmol. 1988;106:629-639.

12. Lewis JM, Park I, Ohji M, Saito Y, Tano Y. Diamond-dusted silicone cannula for epiretinal membrane separation during vitreous surgery. Am J Ophthalmol. 1997;124:552-554.

13. Sakaguchi H, Ikuno Y, Choi JS, Ohji M, Tano T. Multiple components of epiretinal tissues detected by triamcinolone and indocyanine green in macular hole and retinal detachment as a result of high myopia. Am J Ophthalmol. 2004;138:1079-1081.

14. Cherfan GM, Michels RG, de Bustros S, Enger C, Glaser BM. Nuclear sclerotic cataract after vitrectomy for idiopathic epiretinal membranes causing macular pucker. Am J Ophthalmol. 1991;111:434-438.

15. Smiddy WE, Feuer W. Incidence of cataract extraction after diabetic vitrectomy. Retina. 2004;24:574-581.

16. Ezra E, Gregor ZJ. Surgery for idiopathic full-thickness macular hole: two-year results of a randomized clinical trial comparing natural history, vitrectomy, and vitrectomy plus autologous serum: Moorfields Macular Hole Study Group Report No 1. Arch Ophthalmol. 2004;122: 224-236.

17. Wakabayashi T, Fujiwara M, Sakaguchi H, Kusaka S, Oshima Y. Foveal microstructure and visual acuity in surgically closed macular holes: spectral-domain optical coherence tomographic analysis. Ophthalmology. 2010;117:1815-1824.

18. Wittich W, Overbury O, Kapusta MA, Watanabe DH. Hierarchical linear modeling of visual acuity change over time: rate of functional recovery after macular hole surgery. Optom Vis Sci. 2007;84:872-878.

19. Wendel RT, Patel AC, Kelly NE, Salzano TC, Wells JW, Novack GD. Vitreous surgery for macular holes. Ophthalmology. 1993;100: 1671-1676.

20. Kwok AK, Lai TY, Li WW, Woo DC, Chan NR. Indocyanine greenassisted internal limiting membrane removal in epiretinal membrane surgery: a clinical and histologic study. Am J Ophthalmol. 2004;138: 194-199.
Clinical Ophthalmology

\section{Publish your work in this journal}

Clinical Ophthalmology is an international, peer-reviewed journal covering all subspecialties within ophthalmology. Key topics include: Optometry; Visual science; Pharmacology and drug therapy in eye diseases; Basic Sciences; Primary and Secondary eye care; Patient Safety and Quality of Care Improvements. This journal is indexed on Submit your manuscript here: http://www.dovepress.com/clinical-ophthalmology-journal

\section{Dovepress}

PubMed Central and CAS, and is the official journal of The Society of Clinical Ophthalmology (SCO). The manuscript management system is completely online and includes a very quick and fair peer-review system, which is all easy to use. Visit http://www.dovepress.com/ testimonials.php to read real quotes from published authors. 